\title{
THE ASYMPTOTIC DIMENSION OF A CURVE GRAPH IS FINITE
}

\author{
GREGORY BELL AND KOJI FUJIWARA \\ Dedicated to Professor Yukio Matsumoto on his 60th birthday.
}

\begin{abstract}
We find an upper bound for the asymptotic dimension of a hyperbolic metric space with a set of geodesics satisfying a certain boundedness condition studied by Bowditch. The primary example is a collection of tight geodesics on the curve graph of a compact orientable surface. We use this to conclude that a curve graph has finite asymptotic dimension. It follows then that a curve graph has property $A_{1}$. We also compute the asymptotic dimension of mapping class groups of orientable surfaces with genus $\leq 2$.
\end{abstract}

\section{INTRODUCTION}

The asymptotic dimension of a metric space was defined by Gromov 22, page 29] as the large-scale analog of Lebesgue covering dimension. Gromov originally denoted asymptotic dimension asdim $_{+}$, but the notation asdim has become more standard. Define asdim $X \leq n$ if for every (large) $r>0$ there is a cover of $X$ by uniformly bounded subsets of $X$ so that no ball of radius $r$ in $X$ meets more than $n+1$ elements of the cover, (see section 2). Asymptotic dimension is a coarse invariant (see section 2), so in particular it is a quasi-isometry invariant.

The notion of a $\delta$-hyperbolic space is due to Gromov 21] and has been studied extensively. Gromov remarked [22, page 31] that finitely generated hyperbolic groups have finite asymptotic dimension. Recently Roe 40 improved on this result, showing that a hyperbolic geodesic metric space with bounded growth (see section 2 ) has finite asymptotic dimension. This also follows from a result of Bonk and Schramm 8].

Below we consider the asymptotic dimension of hyperbolic geodesic graphs which need not have bounded growth, but satisfy a boundedness condition in terms of geodesics that we call property $B$. Although the property is stated locally, it is indeed a global condition. A simple example of a space with property B that does not have bounded growth is an infinite-valence tree. In Theorem 1 we prove that hyperbolic spaces with property B have finite asymptotic dimension.

Let $S=S_{g, p}$ denote a compact, orientable surface of genus $g$ with $p$ punctures (for technical reasons, we require $3 g-3+p>1$ ). The complex of curves, $C(S)$ was defined by Harvey 25] in his study of Teichmüller spaces of Riemannian manifolds. It is a simplicial complex whose vertices are isotopy classes of essential curves on $S$; a collection of vertices span a simplex when they can be realized simultaneously by disjoint curves on $S$. The curve graph is the 1-skeleton $C^{(1)}(S)$ and is denoted $X(S)$.

Date: November 1, 2006.

2000 Mathematics Subject Classification. 57M99 (primary), 20F69 (secondary). 
Masur and Minsky [36] showed that $X(S)$ is a hyperbolic graph, (see Theorem 2). Although $X(S)$ does not have bounded growth, Bowditch showed that it does satisfy our boundedness condition, [10. Therefore we can apply Theorem 1 to $X(S)$ to obtain the main result of the first part of the paper:

Corollary 1. Let $S$ be an orientable surface with genus $g$ and $p$ punctures such that $3 g-3+p>1$. Then $\operatorname{asdim} X(S)<\infty$.

A metric space is said to be proper if closed, bounded sets are compact. Although it is interesting to study asymptotic dimension for its own sake, much of the original interest in asymptotic dimension arose in connection with the Novikov conjecture. $\mathrm{G}$. Yu [4] showed that the coarse Baum-Connes conjecture holds for proper metric spaces with finite asymptotic dimension. Applying the so-called descent principle, he concludes that the Novikov conjecture holds for finitely generated groups with finite classifying space and finite asymptotic dimension. This result has since been improved upon by $\mathrm{Yu}[48$ and Kasparov- $\mathrm{Yu}$ [32].

In 48, $\mathrm{Yu}$ defines a property of discrete metric spaces called "property $A$ " guaranteeing the existence of a large-scale (or uniform) embedding into Hilbert space. The existence of this embedding implies the coarse Baum-Connes conjecture for discrete spaces with bounded geometry. As a consequence, finitely generated groups with this property satisfy the Novikov conjecture. Yu's property $A$ is a coarse invariant 48, 45. Many of the examples we consider in this paper are not proper and moreover, they are not even coarsely equivalent to proper metric spaces, so the coarse Baum-Connes conjecture does not apply, (see Proposition 3).

In the fifth section of this paper we show that spaces with our boundedness condition have property $A$ in the sense of Tu 45, which we call property $A_{1}$. This property reduces to Yu's property $A$ when the spaces are discrete with bounded geometry. In particular, we prove:

Corollary 3. Let $S$ be an orientable surface with genus $g$ and $p$ punctures so that $3 g-3+p>1$. Then $X(S)$ has property $A_{1}$.

This follows from Corollary 1 and the proof of a theorem of Higson-Roe [26, Lemma 4.3].

In the sixth section of the paper, we consider the mapping class group of $S$, denoted $\operatorname{Mod}(S)$. By combining a recent result of Dranishnikov [17] with some basic facts about the mapping class groups of surfaces with $g \leq 2$ we can show that $\operatorname{asdim} \operatorname{Mod}(S)=\operatorname{vcd} \operatorname{Mod}(S)$ when $g \leq 2$ and $3 g-3+p>1$. As a corollary, we also find upper bounds for the asymptotic dimension of braid groups, (Corollary 6) and find an exact formula for the asymptotic dimension of some Artin groups, (Corollary 7).

We conclude the paper with a list of open questions about curve complexes and mapping class groups.

Acknowledgements. We wish to thank A. Dranishnikov and G. Yu for many helpful remarks and J. Behrstock, B. Bowditch, D. Margalit, S. Schleimer and M. Kapovich for very helpful comments on a preliminary version of the paper. The second author would like to thank K. Bromberg for a helpful discussion and the Max Planck Institute for Mathematics in Bonn for their hospitality. Finally, the authors would like to thank organizers of the International Conference on Geometric Topology in Będlewo where collaboration for this paper began. 
We also wish to express our very sincere gratitude to the anonymous referee for many helpful comments and a careful reading of the manuscript.

\section{Preliminaries}

There are several equivalent definitions of asymptotic dimension of a metric space (see the survey article [2] or the book [39]). In this paper we will use only the following definition, (mentioned in the introduction) in terms of $r$-multiplicity of a cover. In a metric space $X$, let $N(x ; r)$ denote the $r$-ball centered at $x$ in $X$. Define $\operatorname{asdim} X \leq n$ if for every (large) $r \in \mathbb{N}$ there exists a cover $\left\{U_{i}\right\}_{i}$ of $X$ by uniformly bounded subsets of $X$ so that $\sharp\left\{i \mid N(x ; r) \cap U_{i} \neq \emptyset\right\} \leq n+1$ for all $x \in X$. The number $\sup _{x \in X} \sharp\left\{i \mid N(x ; r) \cap U_{i} \neq \emptyset\right\} \leq n+1$ is called the $r$-multiplicity of the cover $\left\{U_{i}\right\}_{i}$.

A geodesic triangle in a metric space $X$ is said to be $\delta$-thin if each of its sides is contained in the $\delta$-neighborhood of the union of the other two sides. A geodesic space $X$ will be called $\delta$-hyperbolic if every geodesic triangle in $X$ is $\delta$-thin. By a hyperbolic metric space we mean a geodesic metric space which is $\delta$-hyperbolic for some $\delta \geq 0$. We will also say that a finitely generated group is hyperbolic if the Cayley graph with respect to some choice of a finite generating set is $\delta$-hyperbolic for some $\delta \geq 0$. Observe that this property is a quasi-isometry invariant - although the value of $\delta$ is not - so it makes sense to speak of a finitely generated group as hyperbolic without reference to a metric.

A subset $A$ of a metric space $X$ is $r$-discrete if $d\left(a, a^{\prime}\right) \geq r$ for all $a \neq a^{\prime}$ in $A$. The $r$-capacity of a set $Y \subset X$ is the maximal cardinality of an $r$-discrete set in $Y$. A metric space $X$ has bounded geometry if there is an $r>0$ and a function $c:[0, \infty) \rightarrow[0, \infty)$ so that for any $x \in X$ the $r$-capacity of $N(x ; R)$ does not exceed $c(R)$. For a discrete metric space, this simply means that the cardinality of any $R$-ball is bounded by $c(R)$.

A metric space $X$ has bounded growth at some scale if there are constants $r$ and $R$ with $R>r>0$, and $N \in \mathbb{N}$ such that every open ball of radius $R$ in $X$ can be covered by $N$ open balls of radius $r$. A metric space with bounded geometry has bounded growth: take $r$ from the definition of bounded geometry, take $R>r$ and put $N=c(R)$. Clearly the Cayley graph of a finitely generated group has bounded geometry, and so has bounded growth. Thus, any finitely generated group has bounded growth at some scale.

We mentioned that asymptotic dimension is a quasi-isometry invariant, but we will need a stronger statement. It is also a coarse invariant 39. Let $X$ and $Y$ be metric spaces. A map $f: X \rightarrow Y$ is bornologous if for every $R>0$ there is an $S>0$ such that $d\left(f(x), f\left(x^{\prime}\right)\right)<S$ whenever $d\left(x, x^{\prime}\right)<R$. The map $f$ is metrically proper if $f^{-1}(B)$ has bounded diameter in $X$ for each bounded subset $B \subset Y$. A coarse map is a map $f: X \rightarrow Y$ that is bornologous and metrically proper. A coarse map $f: X \rightarrow Y$ is a coarse equivalence between $X$ and $Y$ if there is a coarse map $g: Y \rightarrow X$ and a constant $K \geq 0$ so that $d(f g(y), y) \leq K$ and $d(g f(x), x) \leq K$ for all $x \in X$ and $y \in Y$. It is worth mentioning that in the case that $X$ and $Y$ are finitely generated groups - or indeed any length metric spaces - the notions of coarse equivalence and quasi-isometry coincide, see [39. 


\section{BoundednESS CONDITION}

Let $\Gamma$ be a $\delta$-hyperbolic graph. Suppose that $\mathcal{L}$ is a set of geodesics in $\Gamma$ such that for any $a, b \in V(\Gamma)$, there exists $\gamma \in \mathcal{L}$ connecting $a$ and $b$.

For $a, b \in V(\Gamma)$, let $\mathcal{L}(a, b)$ be the set of all geodesics in $\mathcal{L}$ connecting $a$ and $b$. We write $G(a, b)=\cup \mathcal{L}(a, b) \subset \Gamma$. Given $A, B \subset V(\Gamma)$, let $\mathcal{L}(A, B)=\cup\{\mathcal{L}(a, b)$ $a \in A, b \in B\}$ and $G(A, B)=\cup \mathcal{L}(A, B)$. We write $G(a, b ; r)$ to mean the set $G(N(a ; r), N(b ; r))$.

In [10], Bowditch considered the following boundedness property, which we call property $B$ : there are constants $\ell, k, D$ such that if $a, b \in V(\Gamma), r \in \mathbb{N}$ and $c \in G(a, b)$ with $d(c,\{a, b\}) \geq r+\ell$, then $G(a, b ; r) \cap N(c ; k)$ has at most $D$ elements. We also assume that any geodesic connecting $N(a ; r)$ to $N(b ; r)$ must intersect $N(c ; k)$.

We remark that if this property is satisfied, then we can assume that $k=2 \delta$ because $\Gamma$ is $\delta$-hyperbolic.

We say that a space $X$ has property $B$ if there is a collection $\mathcal{L}$ of geodesics on the space with the above properties.

Theorem 1. Let $\Gamma$ be a $\delta$-hyperbolic graph. Suppose $\mathcal{L}$ is a set of geodesics in $\Gamma$ such that any two vertices of $\Gamma$ are joined by a geodesic in $\mathcal{L}$. If $\mathcal{L}$ satisfies property $B$ with constants $\ell, k=2 \delta$ and $D$, then the asymptotic dimension of $\Gamma$ is at most $2 D-1$.

Proof. Suppose $r \in \mathbb{N}$ is given. We may assume that $\ell \in \mathbb{N}$, and $\ell \geq 10 \delta$. Fix $x_{0} \in \Gamma$. For each $n \in \mathbb{N}$, define

$$
A_{n}=\left\{x \in \Gamma \mid 10(n-1)(r+\ell) \leq d\left(x, x_{0}\right) \leq 10 n(r+\ell)\right\} .
$$

Clearly $\cup_{n} A_{n}=\Gamma$. Let

$$
S_{n}=\left\{x \in \Gamma \mid d\left(x, x_{0}\right)=10 n(r+\ell)\right\} .
$$

For each $n \geq 3$, we define subsets $\left\{U_{i}^{n}\right\}_{i}$ of $A_{n}$ such that $\cup_{i} U_{i}^{n}=A_{n}$ as follows. Write out the elements of $S_{n-2}$ as $\left\{s_{i}\right\}$. Define

$$
U_{i}^{n}=\left\{\left.x \in A_{n}\right|^{\exists}\left[x, x_{0}\right] \in \mathcal{L} \text { s.t. } s_{i} \in\left[x, x_{0}\right]\right\},
$$

where $\left[x, x_{0}\right]$ is a geodesic from $x$ to $x_{0}$. If $n=1$ or 2 , let $U_{1}^{n}=A_{n}$. Clearly, $\cup_{i} U_{i}^{n}=A_{n}$ for each $n$, so the collection $\left\{U_{i}^{n}\right\}_{i, n}$ covers $\Gamma$.

Claim: (1) for any $i$ and for each $n$, $\operatorname{diam} U_{i}^{n} \leq 40(r+\ell)$; and

(2) for an $r / 2$-ball $V$ in $\Gamma$ and for each $n$, there are at most $D$ subsets $U_{i}^{n}$ such that $U_{i}^{n} \cap V \neq \emptyset$.

To see (1), suppose $x, y \in U_{i}^{n}$. If $n=1$ or 2 this is clear, so suppose $n \geq 3$. Then, $d\left(x, s_{i}\right) \leq 20(r+\ell)$, and $d\left(y, s_{i}\right) \leq 20(r+\ell)$, so that $d(x, y) \leq 40(r+\ell)$.

For (2), we observe that this is clear if $n=1$ or 2 , so suppose $n \geq 3$. Suppose $U_{i}^{n} \cap V \neq \emptyset$ and $U_{j}^{n} \cap V \neq \emptyset$. Choose $y_{i} \in U_{i}^{n} \cap V, y_{j} \in U_{j}^{n} \cap V$. Then, $d\left(y_{i}, y_{j}\right) \leq r$, so that $y_{j} \in N\left(y_{i}, r\right)$. Therefore, $s_{j} \in G\left(y_{i}, x_{0} ; r\right)$, because $s_{j} \in\left[x_{0}, y_{j}\right] \in \mathcal{L}$. By the $\delta$-hyperbolicity of $\Gamma, s_{j} \in N\left(s_{i} ; 2 \delta\right)$, since $d\left(y_{i}, s_{i}\right) \geq r+\ell$ and $\ell \geq 10 \delta$. We find $s_{j} \in G\left(y_{i}, x_{0} ; r\right) \cap N\left(s_{i} ; 2 \delta\right)$. Fixing $i$ and allowing $j$ to vary we see, by property $\mathrm{B}$ (letting $x_{0}=a, y_{i}=b, s_{i}=c$ ), that there are at most $D$ such $s_{j}$ satisfying this property since $d\left(x_{0}, s_{i}\right) \geq r+\ell$. So we have the claim.

It follows that the collection $\left\{U_{i}^{n}\right\}_{i, n}$ is a uniformly bounded cover with $r / 2$ multiplicity $\leq 2 D$. The last item is clear from claim (2) because for any $x \in \Gamma$ there can be at most two sets of the form $A_{n}$ with $A_{n} \cap N(x ; r / 2) \neq \emptyset$. Thus, $\operatorname{asdim} \Gamma \leq 2 D-1$ as required. 
Examples. (1) Let $T$ be a tree, which is 0-hyperbolic. Let $\mathcal{L}$ be the set of all geodesics in $T . \mathcal{L}$ satisfies the property B with $D=1, k=0, \ell=0$, so that the asymptotic dimension of $T$ is at most 1 . We should note that this was known to Gromov [22, 1.E., Example (b)] and an explicit proof appears in Roe's book 39 .

(2) Let $\Gamma$ be the Cayley graph of a word-hyperbolic group $G$ with respect to a finite generating set, $S$, with $S^{-1}=S$. Let $|S|=s$. Let $\mathcal{L}$ be the set of all geodesics in $\Gamma$. Any two points are joined by a geodesic. Suppose that $\Gamma$ is $\delta$-hyperbolic. For a point $a \in V(\Gamma)$, let $D=|N(a ; 2 \delta)| \leq s^{2 \delta}$. Note that $D$ does not depend on $a$ since $G$ acts transitively on $\Gamma$ by isometries. Then, $\mathcal{L}$ satisfies property B with $k=2 \delta, \ell=10 \delta$ and $D$. Thus, we conclude that $\operatorname{asdim} \Gamma \leq 2 s^{2 \delta}-1$.

\section{Curve Graph}

Let $S=S_{g, p}$ be a compact orientable surface of genus $g$ with $p$ punctures. The curve complex of $S$ was defined by Harvey 25] and has been successfully used in the study of mapping class groups, for example in [4, 24, 29]. For a good overview we recommend Ivanov 28. For our purposes, we will restrict attention to the 1skeleton of the curve complex, called the curve graph. The curve graph $X$ of $S$ is a graph whose vertices are isotopy classes of essential, nonparallel, nonperipheral, simple closed curves in $S$ where two distinct vertices are joined by an edge if the corresponding curves can be realized simultaneously by pairwise disjoint curves. In certain sporadic cases $X$ as defined above is 0-dimensional or empty. This happens when there are no curve systems consisting of two curves, i.e. $3 g-3+p \leq 1$, (see the discussion following the Remark (ii) below). In the discussion that follows these cases are excluded. The mapping class group $\operatorname{Mod}(S)$ of $S$ (see Section 6) acts on $X$ by $f \cdot a=f(a)$. H. Masur and Y. Minsky proved the following remarkable result.

Theorem 2 (36]). Let $S$ be a compact orientable surface of genus $g$ with $p$ punctures. Suppose $3 g-3+p>1$. Then the curve graph $X(S)$ is hyperbolic.

It is known (see [36]) that $X$ is connected, locally infinite, and its diameter is infinite. In general there are infinitely many geodesics connecting $a, b \in X$; however, Masur-Minsky found a set, $\mathcal{L}$, of geodesics, called tight geodesics such that $\mathcal{L}(a, b)$ is not empty and finite for any $a, b \in X$.

Bowditch showed the following.

Theorem 3. 10, Theorem 1.2] Let $X$ denote the curve graph of a surface $S_{g, p}$ with $3 g-3+p>1$. Let $\mathcal{L}$ be the set of tight geodesics on $X$. Then $\mathcal{L}$ satisfies property $B$ for some $k, \ell$ and $D$.

It follows from Theorem 1 that the asymptotic dimension of the curve graph $X$ is at most $2 D-1$ so in particular it is finite.

Corollary 1. Let $S_{g, p}$ be an orientable surface of genus $g$ with $p$ punctures such that $3 g-3+p>1$. Then $\operatorname{asdim} X\left(S_{g, p}\right)<\infty$.

Remarks. (1) As we said, the curve graph of $S$ is the 1-skeleton of the curve complex of $S$. They are quasi-isometric, so they have same asymptotic dimension. 
(2) After this paper was written, Fujiwara and Whyte [20] showed that a hyperbolic geodesic space with asymptotic dimension 1 is quasi-isometric to a tree. Using results of Behrstock and Leininger, Schleimer [41] showed the following property for $X=X\left(S_{g, 1}\right)$ with $g \geq 2$ : for any $x \in X$ and $r \in \mathbb{N}$, $X \backslash N(x ; r)$ is connected. It follows that $X$ is not coarsely equivalent to a tree (cf. Proposition 3). This implies that asdim $X\left(S_{g, 1}\right)>1(g \geq 2)$, 20]. Thus there exist surfaces whose curve graphs have asymptotic dimension greater than 1. It is unclear what happens in other cases.

As mentioned above, when $3 g-3+p \leq 1$ the curve graph as defined above is 0 -dimensional or empty. In these cases, one could rectify the situation by declaring two vertices to be joined by an edge if the corresponding curves can be realized with only one intersection point. One such case of particular interest is the once-punctured torus $(g=1, p=1)$. By placing an edge between curves if their intersection number is 1, we obtain a Farey graph. It is the 1-skeleton of a planar 2-complex whose dual tree is a binary tree.

A Farey graph $X$ (or the Farey graph since they are all isomorphic) can also be described as follows (see, for example [38, §3], which provides a nice picture of $X$ ). In the rational projective line $\hat{\mathbb{Q}}=\mathbb{Q} \cup\{\infty\}$ identify 0 with $0 / 1$ and $\infty$ with $1 / 0$. The set $\hat{\mathbb{Q}}$ forms the vertex set of $X$. Connect the (reduced) fractions $p / q$ and $r / s$ by an edge precisely when $|p s-r q|=1$. The resulting graph is connected and each edge separates the graph.

The following result appears as Example 5.2 in 35, where Manning uses an interesting condition called the bottleneck property.

Proposition 1 (35). A Farey graph is quasi-isometric to a regular infinite-valence tree.

Applying Example 1 from Section 2, we immediately obtain the following result (see the remarks before Question 3 in Section 6 for the lower bound).

Corollary 2. The asymptotic dimension of a Farey graph is 1 .

From Proposition 1 it is easy to see that a Farey graph is not quasi-isometric to a finite valence tree. (See Proposition 4 for a stronger statement.)

\section{Property $A_{1}$}

G. Yu introduced property $A$ for discrete metric spaces as a means to guarantee the existence of a uniform embedding into Hilbert space [48. The existence of this embedding implies the coarse Baum-Connes conjecture for discrete metric spaces with bounded geometry. (As mentioned in the introduction many of the spaces we consider are not examples of such spaces.) Yu's definition was the following:

Definition. Let $\operatorname{fin}(Z)$ denote the collection of finite, nonempty subsets of $Z$. The discrete metric space $Z$ has property $A$ if there are maps $A_{n}: Z \rightarrow \operatorname{fin}(Z \times \mathbb{N})$ $(n=1,2, \ldots)$ such that

(1) for each $n$ there is some $R>0$ so that

$$
A_{n}(z) \subset\left\{\left(z^{\prime}, j\right) \in Z \times \mathbb{N} \mid d\left(z, z^{\prime}\right)<R\right\}
$$

for every $z \in Z$ and 
(2) for every $K>0$

$$
\lim _{n \rightarrow \infty} \sup _{d(z, w)<K} \frac{\left|A_{n}(z) \Delta A_{n}(w)\right|}{\left|A_{n}(z) \cap A_{n}(w)\right|}=0 .
$$

Following Tu [45] and Dranishnikov [16], define (for $p \in \mathbb{R} \cup+\infty$ ) a metric space $X$ to have property $A_{p}$ if there exist maps $a^{n}: X \rightarrow \ell^{p}(X)$ such that $\left\|a_{x}^{n}\right\|_{p}=1$, $a_{x}^{n}(y) \geq 0$ for all $x, y \in X, n \in \mathbb{N}$, and

(1) there is a function $R=R(n)$ so that for all $x \in X, \operatorname{supp}\left(a_{x}^{n}\right) \subset N(x ; R)$, and

(2) for every $K>0$,

$$
\lim _{n \rightarrow \infty} \sup _{d(x, y)<K}\left\|a_{x}^{n}-a_{y}^{n}\right\|_{p}=0 .
$$

This definition is similar to a characterization of property $A$ for discrete metric spaces with bounded geometry given by Higson and Roe in [26]. There they show that for discrete metric spaces with bounded geometry, property $A_{1}$ is equivalent to Yu's Property $A$. Dranishnikov showed in [16 that discrete metric spaces with property $A_{p}$ admit a coarse embedding in $\ell^{p}$. In [45, Proposition 3.2], Tu shows that property $A_{1}$ and $A_{2}$ are equivalent. The proposition is stated for discrete metric spaces, but the remarks following the proof state that the equivalence holds in general. Also in remarks following this proposition, Tu states that Property $A$ (in the sense of $\mathrm{Yu}$ ) implies property $A_{1}$. On the other hand, it is unknown whether $A_{1}$ implies property $A$ without the requirement of bounded geometry. In [16. Proposition 3.2], Dranishnikov shows that properties $A_{p}$ and $A_{q}$ are equivalent for any $1 \leq p, q<\infty$.

The relation between finite asymptotic dimension and these properties is the following:

Lemma 1. 26, Lemma 4.3] Let $X$ be a discrete metric space with bounded geometry and finite asymptotic dimension. Then $X$ has property $A$.

In fact, they show $X$ has property $A_{1}$, which is equivalent to property $A$ for such spaces. Their argument along with Theorem 1 can be used to show the following result:

Theorem 4. Let $\Gamma$ be a $\delta$-hyperbolic graph. Suppose $\mathcal{L}$ is a set of geodesics in $\Gamma$ such that any two vertices of $\Gamma$ are joined by a geodesic in $\mathcal{L}$. If $\mathcal{L}$ satisfies property $B$ with constants $\ell, k=2 \delta$ and $D$, then $\Gamma$ has property $A_{1}$.

We reproduce the argument from [26] for the reader's convenience. We also fill in some details using our definition of asymptotic dimension in terms of $r$-multiplicity of the cover.

Proof. By Theorem 1, asdim $\Gamma \leq 2 D-1$. By the definition of asdim, we know that for any $r \in \mathbb{N}$ we can find a uniformly bounded cover $\mathcal{U}$ of $\Gamma$ with $5 r$-multiplicity $\leq 2 D$. Define a cover $\mathcal{V}$ of $\Gamma$ by $\mathcal{V}=\{N(U ; 2 r) \mid U \in \mathcal{U}\}$, where $N(U ; 2 r)$ denotes the $2 r$-neighborhood of $U$ in $\Gamma$. Clearly $\mathcal{V}$ covers $\Gamma$ and $\operatorname{diam}(V) \leq 4 r+\operatorname{diam}(U)$, so $\mathcal{V}$ consists of sets with uniformly bounded diameters. Next, if $x \in N(U ; 2 r)$ then $N(x ; 5 r)$ meets $U$. For any $x$ there are at most $2 D$ such $U$, so the multiplicity (or order) of $\mathcal{V}$ is $\leq 2 D$. Finally, let $A \subset \Gamma$ with $\operatorname{diam}(A)<r$. If $x \in A$, then $A \subset N(U ; 2 r)$ where $U \in \mathcal{U}$ is any set containing $x$. Thus, any set with diameter 
$<r$ is entirely contained within some element of the cover $\mathcal{V}$. (This says exactly that $r$ is a Lebesgue number for $\mathcal{V}$.)

For each $V \in \mathcal{V}$, define a map $\phi_{V}^{r}: \Gamma \rightarrow[0,1]$ by

$$
\phi_{V}^{r}(x)=\frac{d(x, \bar{V})}{\sum_{W \in \mathcal{V}} d(x, \bar{W})},
$$

where the bar denotes the complement in $\Gamma$. Clearly, each $\phi_{V}^{r} \not \equiv 0$. For each $V \in \mathcal{V}$ let $x_{V}$ denote some point with $\phi_{V}^{r}\left(x_{V}\right) \neq 0$. Now, define $a^{r}: \Gamma \rightarrow \ell^{1}(\Gamma)$ by

$$
a_{x}^{r}(z)=\sum_{V \in \mathcal{V}} \phi_{V}^{r}(x) \delta_{x_{V}}(z),
$$

where $\delta_{x_{V}}$ is the Dirac- $\delta$ function. Clearly $a_{x}^{r}$ assumes a nonzero value at no more than $2 D$ points in $\Gamma$ and so $a_{x}^{r} \in \ell^{1}(\Gamma)$.

\section{Claim:}

(1) $a_{x}^{r}(z) \geq 0$ for all $z \in \Gamma$;

(2) $\left\|a_{x}^{r}\right\|_{1}=1$;

(3) there is some $R=R(r)$ so that $\operatorname{supp}\left(a_{x}^{r}\right) \subset N(x ; R)$ for all $x \in \Gamma$; and

(4) for every $K>0$

$$
\lim _{r \rightarrow \infty} \sup _{d(z, w)<K}\left\|a_{z}^{r}-a_{w}^{r}\right\|_{1}=0 .
$$

Item (1) is clear. For item (2), we compute

$$
\begin{aligned}
\left\|a_{x}^{r}\right\|_{1} & =\sum_{z \in \Gamma}\left|\sum_{V \in \mathcal{V}} \phi_{V}^{r}(x) \delta_{x_{V}}(z)\right| \\
& =\sum_{V \in \mathcal{V}} \phi_{V}^{r}(x)=1 .
\end{aligned}
$$

To see item $(3)$, we take $R(r)=4 r+\operatorname{diam}(\mathcal{U})$ where $\operatorname{diam}(\mathcal{U})$ is an upper bound for the diameters of the sets in $\mathcal{U}$. Now, $a_{x}^{r}(z)>0$ means that $z=x_{V}$ for some $V$ containing $x$. Thus, $z \in V$, so $d(z, x) \leq R$.

Finally, for item (4), let $K>0$ and $\epsilon>0$ be given. Take $r$ so large that $(4 D+1)^{2} K / r<\epsilon$. The triangle inequality says if $z, w \in \Gamma$ and $V \in \mathcal{V}$, then $|d(z, \bar{V})-d(w, \bar{V})| \leq d(z, w)$. Also, observe that for any $w \in \Gamma, \sum_{U \in \mathcal{V}} d(w, \bar{U}) \geq r$ since $r$ is a Lebesgue number for the cover $\mathcal{V}$. Thus, we have

$$
\begin{gathered}
\left|\phi_{V}^{r}(z)-\phi_{V}^{r}(w)\right|=\left|\frac{d(z, \bar{V})}{\sum_{U \in \mathcal{V}} d(z, \bar{U})}-\frac{d(w, \bar{V})}{\sum_{U \in \mathcal{V}} d(w, \bar{U})}\right| \\
\leq \frac{|d(z, \bar{V})-d(w, \bar{V})|}{\sum_{U \in \mathcal{V}} d(z, \bar{U})}+\left|\frac{d(w, \bar{V})}{\sum_{U \in \mathcal{V}} d(z, \bar{U})}-\frac{d(w, \bar{V})}{\sum_{U \in \mathcal{V}} d(w, \bar{U})}\right| \\
\leq \frac{d(z, w)}{\sum_{U \in \mathcal{V}} d(z, \bar{U})}+\frac{d(w, \bar{V})}{\sum_{U \in \mathcal{V}} d(z, \bar{U}) \sum_{U \in \mathcal{V}} d(w, \bar{U})} \sum_{U \in \mathcal{V}}|d(w, \bar{U})-d(z, \bar{U})| \\
\leq \frac{1}{r} d(z, w)+\frac{1}{r}\left(\sum_{U \in \mathcal{V}}|d(z, \bar{U})-d(w, \bar{U})|\right) \\
\leq \frac{1}{r} d(z, w)+\frac{4 D d(z, w)}{r}=\frac{(4 D+1)}{r} d(z, w) .
\end{gathered}
$$


Then,

$$
\begin{aligned}
\left\|a_{z}^{r}-a_{w}^{r}\right\|_{1} & =\sum_{x \in \Gamma}\left|a_{z}^{r}(x)-a_{w}^{r}(x)\right| \\
& =\sum_{x \in \Gamma}\left|\sum_{V} \phi_{V}^{r}(z) \delta_{x_{V}}(x)-\sum_{V} \phi_{V}^{r}(w) \delta_{x_{V}}(x)\right| \\
& =\left|\sum_{V}\left(\phi_{V}^{r}(z)-\phi_{V}^{r}(w)\right) \delta_{x_{V}}\right| \\
& \leq \sup _{V}\left|\phi_{V}^{r}(z)-\phi_{V}^{r}(w)\right| 4 D \\
& \leq 4 D \frac{(4 D+1)}{r} d(z, w) \leq \frac{(4 D+1)^{2} K}{r}<\epsilon .
\end{aligned}
$$

So item (4) is proved. Thus, $\Gamma$ has property $A_{1}$.

As mentioned in Theorem 3, Masur-Minsky's tight geodesics $\mathcal{L}$ on a curve graph satisfy property B. Thus we have the following result.

Corollary 3. Let $S$ be an orientable surface of genus $g$ with $p$ punctures such that $3 g-3+p>1$. Then the curve graph of $S$ has property $A_{1}$.

$\mathrm{Tu}$ [45, Proposition 8.1] also proved that a discrete hyperbolic metric space $X$ with bounded geometry has property $A_{1}$ by fixing $a \in \partial X$ and taking for the functions collections of geodesic rays marching off to $a$. As it stands, this argument cannot be applied directly to discrete hyperbolic spaces with a collection of geodesics satisfying property $\mathrm{B}$ as the following example shows.

Example. Let $T$ be the following tree. Let $x_{0}$ be a vertex with infinite valence. Issuing from $x_{0}$ take edges of length $1,2,3, \ldots$ Clearly, $T$ is hyperbolic and the collection of all geodesics in $T$ satisfies property $\mathrm{B}$, but $\partial T=\emptyset$, so Tu's argument does not apply.

On the other hand, suppose $\Gamma$ is a discrete hyperbolic space with a collection of geodesics $\mathcal{L}$ with property $\mathrm{B}$ such that any two points in $\Gamma$ are connected by a geodesic in $\mathcal{L}$. If $\Gamma$ has the additional properties that $\partial \Gamma \neq \emptyset$ and that $\gamma \in \mathcal{L}$ implies any subgeodesic of $\gamma$ is also in $\mathcal{L}$, then Tu's argument can be applied with very few changes to show that $\Gamma$ has property $A_{1}$. The point of this remark is that when we follow his argument we use only geodesics in the set $\mathcal{L}$ to deal with the problem that $\Gamma$ may not have bounded geometry. We note that since the set of tight geodesics of a curve graph satisfies these additional properties, this approach gives another argument to show that a curve graph has property $A_{1}$. After this paper was written we were informed that Y. Kida 33 has shown that a curve graph has not only property $A_{1}$ but also property $A$.

A natural question at this point is whether the coarse Baum-Connes conjecture is true for a curve complex. We settle this question by showing that a curve graph is not coarsely equivalent to a proper metric space, so the coarse Baum-Connes conjecture cannot be formulated for it. It is likely that this is well known to specialists, but we do not know a reference, so we record it.

The following lemma is well known.

Lemma 2. Let $f: X \rightarrow Y$ be a coarse equivalence with coarse inverse $g: Y \rightarrow X$. Then, for every $R$ there is an $S$ so that $d\left(x, x^{\prime}\right) \geq S$ implies $d\left(f(x), f\left(x^{\prime}\right)\right) \geq R$.

Proof. Since $X$ and $Y$ are coarsely equivalent, there is some $K>0$ so that $d\left(f g, 1_{Y}\right) \leq K$ and $d\left(g f, 1_{X}\right) \leq K$. Let $R$ be given. Since $g$ is a coarse map, there is a $S_{g}>0$ so that $d\left(y, y^{\prime}\right)<R$ implies that $d\left(g(y), g\left(y^{\prime}\right)\right)<S_{g}$. Put $S>2 S_{g}+2 K$. Then, if $d\left(x, x^{\prime}\right) \geq S$, we have $d\left(g f(x), g f\left(x^{\prime}\right)\right) \geq 2 S_{g}+2 K-2 K$. 
So, if $d\left(f(x), f\left(x^{\prime}\right)\right)<R$, then we have $d\left(g f(x), g f\left(x^{\prime}\right)\right)<S_{g}$, which is a contradiction.

Let $D>0$ be given. By an infinite $D$-discrete bounded subset of $X$ we mean an infinite collection of points $x_{i}$ in $X$ such that $d\left(x_{i}, x_{j}\right) \geq D$ whenever $i \neq j$ and $\sup \left\{d\left(x_{i}, x_{j}\right) \mid i, j\right\}<\infty$.

Proposition 2. Let $X$ be a metric space that has infinite D-discrete bounded subsets for some $D$. Then $X$ is not proper.

Proof. Take a sequence $\left\{x_{i}\right\}$ of distinct points in the infinite $D$-discrete bounded subset. If $X$ were proper, this sequence would have a convergent subsequence. Since $d\left(x_{i}, x_{j}\right) \geq D$ for all $i \neq j$, this cannot happen.

Lemma 3. Having an infinite D-discrete bounded subset for all D is an invariant of coarse isometry.

Proof. Let $f$ and $g$ be coarse maps from $X$ to $Y$ and $Y$ to $X$ that give a coarse equivalence between $X$ and $Y$. Let $D>0$ be given. Choose $D^{\prime}>0$ so that if $d\left(x, x^{\prime}\right) \geq D^{\prime}\left(x, x^{\prime} \in X\right)$ then $d\left(f(x), f\left(x^{\prime}\right)\right) \geq D$. Let $A \subset X$ be an infinite $D^{\prime}$-discrete bounded subset. Then, $f(A) \subset Y$ is $D$-discrete by our choice of $D^{\prime}$ and bounded by the fact that $f$ is bornologous.

Corollary 4. Let $G$ be a Farey graph. Then $G$ is not coarsely equivalent to a proper metric space.

Proof. Let $T$ be a tree with infinite valence. We saw that $G$ and $T$ are quasiisometric. Since a coarse isometry between $G$ and a proper metric space $X$ would yield a coarse isometry between $X$ and $T$, it suffices to show that $T$ and $X$ are not coarsely isometric. So, we need only show that $T$ has infinite $D$-discrete bounded subsets for any $D>0$.

To this end, let $D>0$ be given. Fix a base vertex $t_{0} \in T$ and enumerate the infinitely many vertices $t_{1}, t_{2}, \ldots$ adjacent to $t_{0}$. Let $\gamma_{i}(i=1,2, \ldots)$ be geodesic rays from $t_{0}$ so that $\gamma_{i}$ contains $t_{i}$. Define $A=\left\{\gamma_{i}(D)\right\}$. Now, $A$ is an infinite $2 D$-discrete, $2 D$-bounded subset of $T$. In particular, it is an infinite $D$-discrete bounded subset of $T$.

We remark that the above argument shows that there is no map $f: G \rightarrow X$ such that there exist $C>0$ and $\epsilon \geq 0$ such that for any $x, y \in G, \frac{1}{C} d(x, y)-\epsilon \leq$ $d(f(x), f(y)) \leq C d(x, y)+\epsilon$, (see the remarks following the definition of coarse equivalence in Section 2).

Lemma 4. $X=X\left(S_{g, p}\right), 3 g+p-4>0$, has infinite $D$-discrete bounded subsets for all $D$.

Proof. We use the following theorem by Bowditch [10] concerning certain properness, which he calls acylindricity, of the action of $\operatorname{Mod}(S)$ on $X(S)$ (cf. the property WPD in [4; WPD is weaker, but enough for our purpose).

Theorem 5. 10, Acylindricity] Let $X\left(S_{g, p}\right), 3 g+p-4>0$ be a curve graph. For any $D>0$, there exist $L, K>0$, which depend on $D$ and $S_{g, p}$, with the following property: if $x, y \in X$ are two points with $d(x, y) \geq L$, then there are at most $K$ elements a in $\operatorname{Mod}(S)$ such that $d(x, a x) \leq D$ and $d(y, a y) \leq D$. 
Fix a surface $S$. Suppose $D>0$ is given. Let $L>0, K>0$ be constants from Theorem 5 for $D$ and $S$. Choose two points $x, y \in X$ with $d(x, y) \geq L$. Let $G<\operatorname{Mod}(S)$ be the stabilizer of $x$. $G$ is an infinite subgroup. For each $g \in G$, since $d(g x, g y) \geq L$ there are at most $K$ elements $a \in G$ with $d(g y, a y) \leq D$ because of the acylindricity (note that $d(g x, a x)=0)$. It follows that, since $G$ is infinite, there are infinitely many elements $g_{i}$ in $G$ such that $d\left(g_{i} y, g_{j} y\right)>D(i \neq j)$. Since all points $g_{i} y$ are in $N(x ; d(x, y)+1)$, we have found the required subset.

Proposition 3. A curve graph $X\left(S_{g, p}\right)$, with $3 g+p-4>0$ is not coarsely equivalent to a proper metric space.

Proof. This is clear from Proposition 2, Lemma 3 and Lemma 4.

Proposition 3 was brought into our attention by Misha Kapovich. His argument is different than the one we gave.

\section{MAPPing ClASS GROUPS}

In this section we turn our attention to mapping class groups. The reader is referred to Ivanov's paper [28] for a thorough introduction to mapping class groups. We reproduce many of the definitions and results contained therein for the reader's convenience. Let $S_{g, p}$ denote the compact orientable surface of genus $g$ with $p$ punctures. The mapping class group of $S_{g, p}, \operatorname{Mod}\left(S_{g, p}\right)$, is the group of isotopy classes of orientation-preserving diffeomorphisms $S_{g, p} \rightarrow S_{g, p}$. It is also known by the name modular group, which explains the notation.

Let $\Gamma$ be a finitely generated group. Fixing a (finite) set of generators $S=S^{-1}$ endows $\Gamma$ with a left-invariant word metric defined by $d_{S}(g, h)=\left\|g^{-1} h\right\|_{S}$, where $\left\|g^{-1} h\right\|_{S}$ is the length of the shortest $S$-word presenting the element $g^{-1} h$. Notice that two finite generating sets give rise to quasi-isometric metric spaces, so we define $\operatorname{asdim} \Gamma$ to be the asymptotic dimension of $\left(\Gamma, d_{S}\right)$ where $S=S^{-1}$ is any finite generating set. We will have occasion to consider countable groups that are not finitely generated. J. Smith [42] showed that every countable group could be endowed with a left-invariant proper metric and that all left-invariant proper metrics are coarsely equivalent. Thus, when speaking of countable groups that may not be finitely generated, we will always assume that the group has a proper leftinvariant metric on it. As a consequence, we see that $\operatorname{asdim} H \leq \operatorname{asdim} G$ for any subgroup $H$ of a finitely generated group $G$.

The mapping class group, $\operatorname{Mod}\left(S_{g, p}\right)$ is finitely generated, (in fact it is finitely presented, see [28]). It was recently shown that $\operatorname{Mod}(S)$ is exact [23, 34. As a consequence, the Novikov conjecture holds for $\operatorname{Mod}(S)$ (cf. [43]). A natural question is whether these groups have finite asymptotic dimension (see Question 2 in Section 7).

It is easy to obtain an obvious lower bound on $\operatorname{asdim} \operatorname{Mod}\left(S_{g, p}\right)$, namely

$$
\operatorname{asdim} \operatorname{Mod}\left(S_{g, p}\right) \geq 3 g-3+p \text {. }
$$

Indeed, there are $k=3 g-3+p$ simple closed curves on $S_{g, p}$ and Dehn twists by these curves commute because they are disjoint. Thus, there is a copy of $\mathbb{Z}^{k}$ inside $\operatorname{Mod}\left(S_{g, p}\right)$. Dranishnikov, Keesling and Uspenskij showed that asdim $\mathbb{Z}^{k}=k$ in 19. Finally, if $Y \subset X$ in a metric space $X$, then any uniformly bounded cover of $X$ by sets with $r$-multiplicity $\leq n+1$ will restrict to a uniformly bounded cover 
of $Y$ with $r$-multiplicity $\leq n+1$, so $\operatorname{asdim} Y \leq \operatorname{asdim} X$. Thus, we conclude that $\operatorname{asdim} \operatorname{Mod}\left(S_{g, p}\right) \geq k$.

This lower bound is unlikely to give the exact asymptotic dimension as we now demonstrate.

Observe that the Euler characteristic of $S_{g, p}$ is $2-2 g-p$. The second part of 28, Theorem 2.8.C] states that when $2-2 g-p<0$, the following sequence is exact:

$$
1 \rightarrow \pi_{1}\left(S_{g, p}\right) \rightarrow \operatorname{PMod}\left(S_{g, p+1}\right) \rightarrow \operatorname{PMod}\left(S_{g, p}\right) \rightarrow 1,
$$

where $\operatorname{PMod}(S)$ is the pure mapping class group of $S$, defined as the group of all isotopy classes of all orientation-preserving diffeomorphisms preserving setwise all boundary components of $S$. Since $\operatorname{PMod}(S)$ is the kernel of the action of $\operatorname{Mod}(S)$ on the set of punctures, $\operatorname{PMod}(S) \subset \operatorname{Mod}(S)$ with finite index. Thus, $\operatorname{asdim} \operatorname{PMod}(S)=\operatorname{asdim} \operatorname{Mod}(S)$.

A finitely presented group $\Gamma$ is said to be of type $F P$ if its classifying space $B \Gamma$ is homotopy dominated by a finite complex. A finitely generated group $\Gamma$ is said to be of type VFP if it contains an FP subgroup of finite index.

The following inequality holds for groups $\Gamma$ of type VFP, [17, Cor 4.11]:

$$
\operatorname{vcd} \Gamma \leq \operatorname{asdim} \Gamma \text {. }
$$

In [28, §5.4], Ivanov shows that $\operatorname{Mod}\left(S_{g, p}\right)$ is of type VFP when $2-2 g-p<0$.

Ivanov computes the virtual cohomological dimension of mapping class groups in 28, Theorems 6.4.A, 6.4.B and 6.4.C:

Theorem 6 (28]). The following equalities hold for $S_{g, p}$, the surface of genus $g$ and $p$ boundary components:

$$
\begin{gathered}
\operatorname{vcd} \operatorname{Mod}\left(S_{0, p}\right)= \begin{cases}0, & \text { if } p \leq 3 \\
p-3, & \text { if } p \geq 3 ;\end{cases} \\
\operatorname{vcd} \operatorname{Mod}\left(S_{1, p}\right)= \begin{cases}1, & \text { if } p=0 ; \\
p, & \text { if } p \geq 1 ;\end{cases} \\
\operatorname{vcd} \operatorname{Mod}\left(S_{g, p}\right)= \begin{cases}4 g-5, & \text { if } g \geq 2, p=0 \\
4 g-4+p, & \text { if } g \geq 2, p \geq 1 .\end{cases}
\end{gathered}
$$

Combining Theorem 6 with Dranishnikov's result (1) gives an improved lower bound on $\operatorname{asdim} \operatorname{Mod}\left(S_{g, p}\right)$ in certain cases.

Next, we turn our attention to an upper bound for asdim $\operatorname{Mod}\left(S_{g, p}\right)$. Let $G$ be a finitely generated group and let $1 \rightarrow K \rightarrow G \rightarrow H \rightarrow 1$ be exact. Being the surjective image of $G, H$ is finitely generated. On the other hand, $K$ is countable but not necessarily finitely generated, so we consider any proper left-invariant metric on $K$. For example, we could give $K$ the metric it inherits as a subset of $G$.

Suppose $1 \rightarrow K \rightarrow G \rightarrow H \rightarrow 1$ is exact and $G$ is finitely generated. When $\operatorname{asdim} K$ and $\operatorname{asdim} H$ are both finite, Bell and Dranishnikov prove that asdim $G \leq$ $\operatorname{asdim} H+\operatorname{asdim} K$ in 3 . If we abuse notation slightly, allowing the terms of our inequalities to be infinity, we get a formula

$$
\operatorname{asdim} \operatorname{PMod}\left(S_{g, p+1}\right) \leq \operatorname{asdim} \operatorname{PMod}\left(S_{g, p}\right)+\operatorname{asdim} \pi_{1}\left(S_{g, p}\right),
$$

when $2-2 g-p<0$. Since $\operatorname{asdim} \operatorname{PMod}(S)=\operatorname{asdim} \operatorname{Mod}(S)$, we get

$$
\operatorname{asdim} \operatorname{Mod}\left(S_{g, p+1}\right) \leq \operatorname{asdim} \operatorname{Mod}\left(S_{g, p}\right)+\operatorname{asdim} \pi_{1}\left(S_{g, p}\right),
$$

when $2-2 g-p<0$. Thus, we can apply an inductive argument on the number of punctures of $S$. We begin with an easy computation. 
Lemma 5. Let $S_{g, p}$ denote the compact surface with genus $g$ and $p$ punctures with $g \geq 1$. Then

$$
\operatorname{asdim} \pi_{1}\left(S_{g, p}\right)= \begin{cases}1, & \text { if } p>0 \\ 2, & \text { if } p=0\end{cases}
$$

Proof. Observe first that $\pi_{1}\left(S_{g, p}\right)$ is a free group if $p>0$, so in this case $\operatorname{asdim} \pi_{1}\left(S_{g, p}\right)=$ 1. If $p=0$ and $g>1$, then $\pi_{1}\left(S_{g, 0}\right)$ is quasi-isometric to $\mathbb{H}^{2}$, so asdim $\pi_{1}\left(S_{g, 0}\right)=2$. Finally, $\pi_{1}\left(S_{1,0}\right)=\mathbb{Z} \oplus \mathbb{Z}$ so asdim $\pi_{1}\left(S_{1,0}\right)=2$.

Theorem 7. Let $p \geq 0$ and $2-2 g-p<0$. If $\operatorname{asdim} \operatorname{Mod}\left(S_{g, 0}\right)<\infty$, then

$$
\operatorname{asdim} \operatorname{Mod}\left(S_{g, p}\right) \leq \operatorname{asdim} \operatorname{Mod}\left(S_{g, 0}\right)+p+1 .
$$

In particular, if asdim $\operatorname{Mod}\left(S_{g, 0}\right)<\infty$ then $\operatorname{asdim} \operatorname{Mod}\left(S_{g, p}\right)<\infty$ for $p \geq 0$.

Proof. This follows easily from the lemma. Indeed,

$$
\begin{aligned}
\operatorname{asdim} \operatorname{Mod}\left(S_{g, p}\right) & \leq \operatorname{asdim} \operatorname{Mod}\left(S_{g, p-1}\right)+\operatorname{asdim} \pi_{1}\left(S_{g, p-1}\right) \\
& \vdots \\
& \leq \operatorname{asdim} \operatorname{Mod}\left(S_{g, 0}\right)+\operatorname{asdim} \pi_{1}\left(S_{g, p-1}\right)+\cdots+\operatorname{asdim} \pi_{1}\left(S_{g, 0}\right) \\
& =\operatorname{asdim} \operatorname{Mod}\left(S_{g, 0}\right)+p+1
\end{aligned}
$$

We can say more when $g \leq 1$. Ivanov explains in [28, 9.2] that $\operatorname{Mod}\left(S_{0,4}\right)$ is commensurable with $\operatorname{PSL}(2, \mathbb{Z})$. Since $\operatorname{PSL}(2, \mathbb{Z})$ is quasi-isometric to a tree, Example (i) in Section 3 implies that asdim $\operatorname{Mod}\left(S_{0,4}\right)=1$. Ivanov also explains that $\operatorname{Mod}\left(S_{1,1}\right) \cong S L(2, \mathbb{Z}) \cong \mathbb{Z}_{4} *_{\mathbb{Z}_{2}} \mathbb{Z}_{6}$, and so asdim $\operatorname{Mod}\left(S_{1,1}\right)=1$. Thus we obtain upper bounds for $\operatorname{asdim} \operatorname{Mod}\left(S_{g, p}\right)$ for $g=0$ or 1 . The lower bounds follow from Theorem 6 and Dranishnikov's estimate (1); combining these bounds gives the following equalities.

Corollary 5. If $p \geq 4$ then

$$
\operatorname{asdim} \operatorname{Mod}\left(S_{0, p}\right)=p-3
$$

and for $p \geq 1$,

$$
\operatorname{asdim} \operatorname{Mod}\left(S_{1, p}\right)=p
$$

Since the braid group $B_{n}$ is isomorphic to the mapping class group of a disk with $n$ punctures, we see that a copy of $B_{n}$ sits inside $\operatorname{Mod}\left(S_{0, n+1}\right)$, the mapping class group of the sphere with $n+1$ punctures. Applying the previous result, we obtain the following.

Corollary 6. Let $B_{n}$ be the braid group on $n$ strands. If $n \geq 3$, $\operatorname{asdim} B_{n} \leq n-2$, so in particular asdim $B_{n}<\infty$ and $B_{n}$ has property $A$.

Let $S$ be a finite set. A Coxeter matrix is a symmetric function $M: S \times S \rightarrow$ $\{1,2,3, \ldots\} \cup\{\infty\}$ with $m(s, s)=1$ and $m\left(s, s^{\prime}\right)=m\left(s^{\prime}, s\right) \geq 2$ if $s \neq s^{\prime}$. The corresponding Coxeter group $\mathcal{W}(M)$ is the group with presentation

$$
\mathcal{W}(M)=\left\langle S \mid\left(s s^{\prime}\right)^{m\left(s, s^{\prime}\right)}=1\right\rangle
$$

where $m\left(s, s^{\prime}\right)=\infty$ means no relation. The associated Artin group $\mathcal{A}(M)$ is the group with presentation

$$
\mathcal{A}(M)=\left\langle S \mid\left(s s^{\prime}\right)^{m\left(s, s^{\prime}\right)}=\left(s^{\prime} s\right)^{m\left(s, s^{\prime}\right)}\right\rangle .
$$


An Artin group with associated Coxeter matrix $M, \mathcal{A}=\mathcal{A}(M)$, is said to be of finite type if $\mathcal{W}(M)$ is finite. It is said to be of affine type if $\mathcal{W}(M)$ acts as a proper, cocompact group of isometries on some Euclidean space with the elements of $S$ acting as affine reflections.

In [18, it was shown that asdim $\mathcal{W}<\infty$ for a Coxeter group $\mathcal{W}$. The following approach to finding an upper bound for the asdim of (certain) Artin groups was suggested by Robert Bell.

In [15], Charney and Crisp observe that each of the Artin groups $\mathcal{A}\left(A_{n}\right), \mathcal{A}\left(B_{n}\right)$ of finite type and the Artin groups $\mathcal{A}\left(\tilde{A}_{n-1}\right)$ and $\mathcal{A}\left(\tilde{C}_{n-1}\right)$ of affine type is a central extension of a finite index subgroup of $\operatorname{Mod}\left(S_{0, n+2}\right)$ when $n \geq 3$. Combining this with the fact that the centers of the Artin groups of finite type are infinite cyclic and the centers of those of affine type are trivial gives the following corollary.

Corollary 7. Let $n \geq 3$. Then if $\mathcal{A}$ is an Artin group of finite type $A_{n}$ or $B_{n}=$ $C_{n}$, we have $\operatorname{asdim} \mathcal{A} \leq n$; if $\mathcal{A}$ is an Artin group of affine type $\tilde{A}_{n-1}$ or $\tilde{C}_{n-1}$, $\operatorname{asdim} \mathcal{A}=n-1$.

Proof. By Corollary 5, asdim $\operatorname{Mod}\left(S_{0, n+2}\right)=n-1$. Since their centers are trivial, $\mathcal{A}\left(\tilde{A}_{n-1}\right)$ and $\mathcal{A}\left(\tilde{C}_{n-1}\right)$ are themselves finite index subgroups of $\operatorname{Mod}\left(S_{0, n+2}\right)$, we conclude that asdim $\mathcal{A}\left(\tilde{A}_{n-1}\right)=n-1$ and $\operatorname{asdim} \mathcal{A}\left(\tilde{C}_{n-1}\right)=n-1$. For the Artin groups of finite type the centers are infinite cyclic. Thus, $\mathcal{A}\left(A_{n}\right) / Z$ and $\mathcal{A}\left(B_{n}\right) / Z$ are finite index subgroups of $\operatorname{Mod}\left(S_{0, n+2}\right)$, so $\operatorname{asdim} \mathcal{A}\left(A_{n}\right) / Z=n-1$, and asdim $\mathcal{A}\left(B_{n}\right) / Z=n-1$. Since asdim of an infinite cyclic group is 1 , the extension theorem for asdim from [3] implies asdim $\mathcal{A}\left(A_{n}\right) \leq n$ and $\operatorname{asdim} \mathcal{A}\left(B_{n}\right) \leq n$.

Corollary 8. By Lemma 1, these groups have property A.

Dan Margalit suggested the following to get an exact formula for the asdim of the mapping class group of genus-2 surfaces. Let $\operatorname{Mod}_{2}\left(S_{g, 0}\right)$ denote the elements of $\operatorname{Mod}\left(S_{g, 0}\right)$ that commute with the hyperelliptic involution, (see [5, 7]). Thinking of $\operatorname{Mod}_{2}\left(S_{g, 0}\right) \subset \operatorname{Mod}\left(S_{g, 0}\right)$, we see that $\operatorname{asdim} \operatorname{Mod}_{2}\left(S_{g, 0}\right) \leq \operatorname{asdim} \operatorname{Mod}\left(S_{g, 0}\right)$. In the genus-2 case, it is true that $\operatorname{Mod}_{2}\left(S_{2,0}\right)=\operatorname{Mod}\left(S_{2,0}\right)$, (see [5, Proposition 3.2] or [7]).

Lemma 6. [5, 28, The following sequence is exact:

$$
1 \rightarrow \mathbb{Z} / 2 \mathbb{Z} \rightarrow \operatorname{Mod}_{2}\left(S_{2,0}\right) \rightarrow \operatorname{Mod}\left(S_{0,6}\right) \rightarrow 1 .
$$

Corollary 9. We have the following formula for the asymptotic dimension of $\operatorname{Mod}\left(S_{2, p}\right)$ :

$$
\operatorname{asdim} \operatorname{Mod}\left(S_{2, p}\right)= \begin{cases}3, & \text { if } p=0 \\ p+4, & \text { if } p>0\end{cases}
$$

Proof. Obviously, asdim $\mathbb{Z} / 2 \mathbb{Z}=0$. By Corollary 5 we have $\operatorname{asdim} \operatorname{Mod}\left(S_{0,6}\right)=$ $6-3=3$. Applying the extension theorem for asdim from 3 to the exact sequence in the previous lemma we see that $\operatorname{asdim} \operatorname{Mod}_{2}\left(S_{2,0}\right) \leq 3$. Thus, $\operatorname{asdim} \operatorname{Mod}\left(S_{2,0}\right) \leq 3$. Finally, Theorem 7 implies that $\operatorname{asdim} \operatorname{Mod}\left(S_{2, p}\right) \leq p+1+3=p+4$. The lower bounds follow from Theorem $[$ and Dranishnikov's estimate (11).

It is interesting to observe that putting our lower bound for $\operatorname{asdim} \operatorname{Mod}\left(S_{g, p}\right)$ together with Theorem 7 we get

$$
4 g-4+p \leq \operatorname{asdim} \operatorname{Mod}\left(S_{g, p}\right) \leq \operatorname{asdim} \operatorname{Mod}\left(S_{g, 0}\right)+1+p,
$$


(when $2-2 g-p<0$ ) so that if $\operatorname{asdim} \operatorname{Mod}\left(S_{g, 0}\right)$ is finite, then $\operatorname{asdim} \operatorname{Mod}\left(S_{g, p}\right)$ increases like the number of punctures.

Lemma 7. Suppose $1 \rightarrow K \stackrel{\iota}{\rightarrow} G \stackrel{\phi}{\rightarrow} H \rightarrow 1$ is an exact sequence of groups with $G$ finitely generated. Suppose asdim $H<\infty$ and $K$ has property $A$ with respect to some (hence any) proper, left-invariant metric. Then $G$ has property $A$.

Proof. Take a finite, symmetric generating set, $S=S^{-1}$, for $G$ and consider the word metric $d_{S}$ on $G$. Taking the word metric $d_{\phi(S)}$ corresponding to the generating set $\phi(S)$ on $H$ implies that $\phi$ is 1-Lipschitz. Let $G$ act on $H$ by the rule $g . h=\phi(g) h$. In [1, the first author showed that if a finitely generated group $G$ acts on a group $H$ with finite asymptotic dimension so that for every $R$, the set $W_{R}=\{g \in G \mid$ $\left.d_{\phi(S)}(g . e, e) \leq R\right\}$ has property $A$, then $G$ has property $A$.

We will prove that $W_{R}=N(\iota(K) ; R)$, where the $R$-neighborhood is taken in $G$, with respect to the word metric in $G$. Thus, $W_{R}$ is quasi-isometric to $\iota(K)$. Thus $W_{R}$ has property $A$ if $\iota(K)$ does. Since $K$ is countable, the metric space $\iota(K)$ and the group $K$ with its given proper metric are coarsely equivalent. Since property $A$ is an invariant of coarse equivalence, this will say that $W_{R}$ has property $A$ for every $R$, and hence, that $G$ has property $A$.

First assume $g \in W_{R}$. Then $\|\phi(g)\|_{\phi(S)} \leq R$. So, there exist $s_{1}, \ldots, s_{k} \in S$, with $k \leq R$, so that $\phi(g)=\phi\left(s_{1}\right) \cdots \phi\left(s_{k}\right)$. Take $g^{\prime}=s_{k}^{-1} \cdots s_{1}^{-1} \in G$. Then, $g g^{\prime} \in K$, and $d\left(g g^{\prime}, g\right) \leq R$. On the other hand, if $g \in N(K ; R)$, then there is a $k \in K$ with $d(g, k) \leq R$. Since $\phi$ is 1 -Lipschitz, we have $d(\phi(g), e) \leq R$, as required.

The Torelli group $\mathcal{I}_{g}$ is the subgroup of $\operatorname{Mod}\left(S_{g, 0}\right)$ acting trivially on $H_{1}\left(S_{g, 0}, \mathbb{Z}\right)$, i.e. the group $\mathcal{I}_{g}$ arising in the following exact sequence:

$$
1 \rightarrow \mathcal{I}_{g} \rightarrow \operatorname{Mod}\left(S_{g, 0}\right) \rightarrow \operatorname{Sp}(2 g, \mathbb{Z}) \rightarrow 1 .
$$

Johnson showed in 31 that $\mathcal{I}_{g}$ is finitely generated if $g \geq 3$, but $\mathcal{I}_{2}$ is non-finitely generated and free, 37. To make sense of the asymptotic dimension of $\mathcal{I}_{2}$, we endow it with a proper left-invariant metric. One such metric on $\mathcal{I}_{2}$ is the one it inherits as a subset of $\operatorname{Mod}\left(S_{2,0}\right)$. Since $\mathcal{I}_{2}$ is isomorphic to a subgroup of $F_{2}$ the free group on two letters, asdim $\mathcal{I}_{2} \leq 1$. Since it contains a bi-infinite geodesic we can conclude that asdim $\mathcal{I}_{2}=1$.

Corollary 10. Endow the Torelli group $\mathcal{I}_{g}$ with a proper (left-invariant) metric. Then $\mathcal{I}_{g}$ has finite asymptotic dimension if and only if $\operatorname{Mod}\left(S_{g, 0}\right)$ does. It has property $A$ if and only if $\operatorname{Mod}\left(S_{g, 0}\right)$ does.

Proof. We showed in Corollary 5 that asdim $\operatorname{Mod}\left(S_{g, 0}\right)<\infty$ when $g<2$ and so these groups (being finitely generated) also have property $A$. For $g<2$ the Torelli groups are trivial so they, too, have both properties.

If $g=2$ we only have to show that $\mathcal{I}_{2}$ has property $A$, but this follows from the corresponding fact for the free group on two generators, which contains it as a subgroup, and the fact that property $A$ is a coarse invariant.

Let $g \geq 3$. In both cases these properties pass to subsets. The exact sequence

$$
1 \rightarrow \mathcal{I}_{g} \rightarrow \operatorname{Mod}\left(S_{g, 0}\right) \rightarrow S p(2 g, \mathbb{Z}) \rightarrow 1
$$

and the fact that asdim is finite for arithmetic groups by 30 implies the result for finite asymptotic dimension. Lemma 7 implies the result for property $A$. 
We briefly discuss Teichmüller spaces. Let $T(S)$ be the Teichmüller space of the surface $S$ with negative Euler characteristic.

See [27] or Chapter 5 in 28] for definitions and information. The group $\operatorname{Mod}(S)$ acts on $T(S)$ naturally. We set $d\left(S_{g, p}\right)=3 g-3+p$. It is known that $T\left(S_{g, p}\right)$ is homeomorphic to $\mathbb{R}^{m}$, where $m=6 g-6+2 p=2 d(S)$. There is a natural metric on $T(S)$, called the Teichmüller metric, which is a Finsler metric. With respect to this metric, $T(S)$ is proper, and the action of $\operatorname{Mod}(S)$ is proper and by isometries. Therefore, we obtain

Proposition 4. Suppose $S$ has negative Euler characteristic and endow $T(S)$ with the Teichmüller metric. Then $\operatorname{asdim} \operatorname{Mod}(S) \leq \operatorname{asdim} T(S)$, where we allow the possibility that the terms of this inequality are infinite.

Proof. The group $\operatorname{Mod}(S)$ acts on $T(S)$ (with the Teichmüller metric) properly by isometries [27, Chapter 6.3], so Proposition 2.3 from [30] applies to this setting. We outline an argument. We fix a point $x \in T(S)$ and identify each element $g \in \operatorname{Mod}(S)$ with $g \cdot x \in T(S)$. We wish to consider the metric on $\operatorname{Mod}(S)$ induced from being a subset in $T(S)$, but this is a pseudo-metric as we could have nontrivial $g$ with $g . x=x$. Since the action is proper, such a $g$ must be torsion, so we may (if necessary) pass to a torsion-free subgroup of $\operatorname{Mod}(S)$ of finite index, 28. This subgroup has the same asdim as $\operatorname{Mod}(S)$. The point is that the metric this group inherits as a subset of $T(S)$ is a coarsely equivalent to a word metric on $\operatorname{Mod}(S)$. Thus, the asymptotic dimension of $\operatorname{Mod}(S)$ as a finitely generated group is the same as the asymptotic dimension of $\operatorname{Mod}(S)$ as a subset of $T(S)$. It follows that $\operatorname{asdim} \operatorname{Mod}(S) \leq \operatorname{asdim} T(S)$.

\section{Some open PROBlems}

Our results lead to some natural questions. Again, we let $S_{g, p}$ be the compact orientable surface of genus $g$ with $p$ punctures and let $X\left(S_{g, p}\right)$ denote its curve graph.

Question 1. What is the asymptotic dimension of $X\left(S_{g, p}\right)$ ?

It is known that $\operatorname{dim}\left(C\left(S_{g, p}\right)\right)=3 g+p-4$ (in the non-exceptional cases where this number is positive). Unfortunately, generally there is no relation between dim and asdim; for instance, $\operatorname{asdim} C(S)=\operatorname{asdim} C^{(1)}(S)$. We showed that $\operatorname{asdim} \operatorname{Mod}\left(S_{g, p}\right)$ (if finite) depends on $g$ and $p$, (see the remarks following Corollary 9). It would be very interesting if $\operatorname{asdim} X\left(S_{g, p}\right)$ were independent of $g$ and $p$.

Perhaps a promising approach to computing the asymptotic dimension of a curve graph would be to examine the boundary $\partial X\left(S_{g, p}\right)$. For finitely generated hyperbolic groups $\Gamma$, it is known that $\operatorname{dim} \partial \Gamma+1=\operatorname{asdim} \Gamma$ by results of Buyalo and Lebedeva [12, cf. [13, 44, 22. In fact, Buyalo and Lebedeva [12] prove this formula not only for groups, but for cobounded, hyperbolic, proper, geodesic metric spaces. The drawback to this approach is that the boundary of $X\left(S_{g, p}\right)$ is not well understood.

If $3 g+p-3>1, X\left(S_{g, p}\right)$ contains a bi-infinite geodesic, (cf. [10]) so asdim $X\left(S_{g, p}\right) \geq$ 1. Note that the asymptotic dimension of a Farey graph is 1, (Corollary 2).

Although we had some finiteness results for $\operatorname{asdim} \operatorname{Mod}\left(S_{g, p}\right)$ the question remains open when $g \geq 3$.

Question 2. Is the asymptotic dimension of $\operatorname{Mod}\left(S_{g, p}\right)$ finite when $g \geq 3$ ? 
Notice that if asdim $\operatorname{Mod}(S)<\infty$ then by Higson and Roe's result 26. Lemma 4.3], $\operatorname{Mod}(S)$ is exact. Thus, the result on the exactness of $\operatorname{Mod}(S)$ 23, 34 would follow from an affirmative answer to this question.

A naive approach to this question would be to attempt to use the Hurewicz-type theorem of Bell and Dranishnikov 1, 3. The $\operatorname{group} \operatorname{Mod}\left(S_{g, p}\right)$ acts on $X\left(S_{g, p}\right)$ by isometries. Since $X\left(S_{g, p}\right)$ has finite asymptotic dimension, we would be able to conclude that $\operatorname{Mod}\left(S_{g, p}\right)$ has finite asymptotic dimension (or property $A$ ) provided we could show that the set $\left\{g \in \operatorname{Mod}\left(S_{g, p}\right) \mid d_{X}\left(g \cdot x_{0}, x_{0}\right) \leq r\right\}$ has finite asymptotic dimension (respectively, property $A$ ) for all $r \in \mathbb{N}$. Here, $x_{0}$ is any point of $X$. We remark that if we regard $x_{0}$ as a curve on $S$ the set we need to analyze contains the stabilizer subgroup of $x_{0}$, which is $\operatorname{Mod}\left(S \backslash x_{0}\right)$. A problem is that those two sets are not necessarily coarsely equivalent (cf. proof of Lemma 7).

L. Ji proved, in [30], that arithmetic groups have finite asymptotic dimension. Ivanov draws a comparison between mapping class groups and arithmetic groups in Chapter 9 of [28. Although the groups are different (cf. [4), many things that are proved true for arithmetic groups are later proved to be true for mapping class groups. So, an affirmative answer to Question 2 does not seem unlikely. Ji gave an upper bound of the asymptotic dimension of an arithmetic group, $\Gamma$, as follows (cf. proof of Proposition 4). Let $X$ be the symmetric space associated to $\Gamma$. Then $\operatorname{asdim} \Gamma \leq \operatorname{asdim} X=\operatorname{dim} X$. So, in connection with Question 2, we ask the following (see Proposition 迁).

Question 3. Let $S=S_{g, p}$ with negative Euler characteristic and endow $T(S)$ with the Teichmüller metric. What is $\operatorname{asdim} T(S)$ ? Is it finite? Is asdim $T(S)$ strictly bigger than $\operatorname{asdim} \operatorname{Mod}(S)$ ? If not (i.e., if they are same), then is $\operatorname{Mod}(S)$ coarsely equivalent to $T(S)$ ?

We can ask similar questions on the asymptotic dimensions of a Teichmüller space with the Weil-Petersson metric (cf.[27]), $g_{\mathrm{WP}}$, but we do not even know if a statement similar to Proposition 4 holds because the space is not proper. Let $n$ be the greatest integer less than or equal to $(d(S)+1) / 2$. Brock and Farb (Theorem 1.4 [11]) proved that there is a quasi-isometric embedding $\mathbb{R}^{n} \rightarrow\left(T\left(S_{g, p}\right), g_{\mathrm{WP}}\right)$. It follows that $\operatorname{asdim}\left(T(S), g_{\mathrm{WP}}\right) \geq n$.

Let $S, S^{\prime}$ be compact orientable surfaces. If there exists a system of disjoint curves, $C$, on $S$ so that one of the connected components of $S \backslash C$ is homeomorphic to the interior of $S^{\prime}$, we write $S^{\prime}<S$. Then we have $\operatorname{asdim}\left(T\left(S^{\prime}\right), g_{\mathrm{WP}}\right) \leq$ $\operatorname{asdim}\left(T(S), g_{\mathrm{WP}}\right)$. This follows from the fact that $\left(T(S), g_{\mathrm{WP}}\right)$ contains a subset which is quasi-isometric to $\left(T\left(S^{\prime}\right), g_{\mathrm{WP}}\right)$. Indeed (cf. [46]), although the metric space $\left(T(S), g_{\mathrm{WP}}\right)$ is not complete, its metric completion $\overline{\left(T(S), g_{\mathrm{WP}}\right)}$ contains $\left(T\left(S^{\prime}\right), g_{\mathrm{WP}}\right)$ as a totally geodesic subspace in such a way that $\left(T\left(S^{\prime}\right), g_{\mathrm{WP}}\right) \subset$ $\overline{\left(T(S), g_{\mathrm{WP}}\right)} \backslash\left(T(S), g_{\mathrm{WP}}\right)$. For $\epsilon>0$, let $N$ be the $\epsilon$-neighborhood of $T\left(S^{\prime}\right)$ in $\overline{T(S)}$. Then $\left(N \backslash T\left(S^{\prime}\right)\right) \subset T(S)$ is quasi-isometric to $T\left(S^{\prime}\right)$.

\section{REFERENCES}

[1] G. Bell, Property A for groups acting on metric spaces, Topology Appl. 130 (2003) 239-251.

[2] G. Bell and A. Dranishnikov, Asymptotic dimension in Będlewo, to appear in Topology Proc., preprint, arXiv:math.GR/0507570 (2005).

[3] G. Bell and A. Dranishnikov, A Hurewicz-type theorem for asymptotic dimension and applications to geometric group theory, Trans. Amer. Math. Soc. 358, (2006), no. 1, 4749-4764. 
[4] M. Bestvina and K. Fujiwara, Bounded cohomology of subgroups of the mapping class groups, Geom. \& Topol. 6 (2002) 69-89.

[5] S. Bigelow and R. Budney, The mapping class group of a genus two surface is linear, Algebr. Geom. Topol. 1 (2001), 699-708.

[6] J. Birman, Braids, links and mapping class groups, Annals of Math. Studies, 82, Princeton University Press, Princeton 1975.

[7] J. Birman and H. Hilden, Lifting and projecting homeomorphisms, Arch. Math. (Basel) 23 (1972), 428-434.

[8] M. Bonk and O. Schramm, Embeddings of Gromov hyperbolic spaces, Geom. Funct. Anal. 10 (2000), no. 2, 266-306.

[9] Brian Bowditch, Intersection numbers and the hyperbolicity of the curve complex, to appear in J. Reine Angew. Math., preprint, www.maths.soton.ac.uk/staff/Bowditch/ (2003).

[10] Brian Bowditch, Tight geodesics in the curve complex, preprint, www.maths.soton.ac.uk/staff/Bowditch/ (2003).

[11] J. Brock, B. Farb, Curvature and rank of Teichmüller space. To appear, American Journal of Mathematics

[12] S. Buyalo and N. Lebedeva, Dimension of locally and asymptotically self-similar spaces, on-line at http://arXiv.org/abs/math.GT/0509433 (2005).

[13] S. Buyalo and V. Schroeder, A product of trees as universal space for hyperbolic groups, on-line at http://arXiv.org/abs/math.GR/0509355 (2005).

[14] G. Carlsson and B. Goldfarb, On homological coherence of discrete groups, J. Algebra 276 (2004), 502-514.

[15] R. Charney and J. Crisp, Automorphism groups of some affine and finite type Artin groups, Math. Res. Lett. 12 (2005), no. 2-3, 321-333.

[16] A. Dranishnikov, Groups with a polynomial dimension growth, Geom. Dedicata 119 (2006), $1-15$.

[17] A. Dranishnikov, Cohomological approach to asymptotic dimension, available on-line at http://arXiv.org/abs/math.MG/0608215. (2006).

[18] A. Dranishnikov and T. Januszkiewicz, Every Coxeter group acts amenably on a compact space, Topology Proc., 24 (1999), 135-141.

[19] A. Dranishnikov, J. Keesling, and V. Uspenskij, On the Higson corona of uniformly contractible spaces, Topology 37 (1998), no. 4, 791-803.

[20] K. Fujiwara and K. Whyte, A note on spaces of asymptotic dimension one, on-line at http://arXiv.org/abs/math.MG/0610391, (2006)

[21] M. Gromov, Hyperbolic groups, Essays in group theory, Math. Sci. Res. Inst. Publ., vol. 8, Springer, 1987, pp. 75-263.

[22] M. Gromov, Asymptotic invariants of infinite groups, Geometric Group Theory, London Math. Soc. Lecture Note Ser. (G. Niblo and M. Roller, eds.), no. 182, 1993.

[23] U. Hamenstädt, Geometry of the mapping class groups I: boundary amenability, on-line at http://arXiv.org/abs/math.GR/0510116/, (2005).

[24] J. L. Harer, The virtual cohomological dimension of the mapping class groups of orientable surfaces, Invent. Math. 84 (1986), 157-176.

[25] W. J. Harvey. Boundary structure of the modular group. In Riemann surfaces and related topics: Proceedings of the 1978 Stony Brook Conference (State Univ. New York, Stony Brook, N.Y ., 1978), pages 245-251, Princeton, N.J., 1981. Princeton Univ. Press.

[26] N. Higson and J. Roe, Amenable group actions and the Novikov conjecture, J. Reine Angew. Math. 519 (2000), 143-153.

[27] Y. Imayoshi, M. Taniguchi, An introduction to Teichmüller spaces. Springer, 1992.

[28] N. V. Ivanov, Mapping class groups, in Handbook of Geometric Topology, (R. Daverman and R. Sher, eds.), 523-633 North-Holland, Amsterdam 2002.

[29] N. V. Ivanov, Automorphisms of complexes of curves and of Teichmüller spaces, Internat. Math. Res. Notices (1997) 651-666.

[30] L. Ji, Asymptotic dimension and the integral $K$-theoretic Novikov conjecture for arithmetic groups, J. Differential Geom. 68 (2004), no. 3, 535-544

[31] D. Johnson, The structure of the Torelli group I: a finite set of generators for $\mathcal{T}$, Annals of Math. 118 (1983) 423-442.

[32] G. Kasparov and G. Yu, The coarse geometric Novikov conjecture and uniform convexity, to appear in Advances in Mathematics (2005). 
[33] Y. Kida, Master's thesis, Kyoto University, 2005.

[34] Y. Kida, Classification of the mapping class groups up to measure equivalence, Proc. Japan Acad., 82, Ser. A, no. 1 (2006), 4ĄE.

[35] J. Manning, Geometry of pseudocharacters, Geom. Topol. 9 (2005) 1147-1185.

[36] Howard A. Masur and Yair N. Minsky. Geometry of the complex of curves. I. Hyperbolicity. Invent. Math., 138 (1999) 103-149.

[37] G. Mess, The Torelli groups for genus 2 and 3 surfaces, Topology 31 (1992), no. 4, 775-790.

[38] Y. Minsky, A geometric approach to the complex of curves on a surface, Topology and Teichmüller spaces (Katinkulta, 1995), 149-158, World Sci. Publ., River Edge, NJ, 1996.

[39] J. Roe, Lectures on coarse geometry, University Lecture Series, vol. 31, AMS, 2003.

[40] J. Roe, Hyperbolic groups have finite asymptotic dimension, Proc. Amer. Math. Soc. 133 (2005), no. 9, 2489-2490.

[41] S. Schleimer, Notes on the complex of curves, available on-line at http://www.math.rutgers.edu/ saulsch/Maths/notes.pdf (2005).

[42] J. Smith, On asymptotic dimension of countable abelian groups, Topology Appl. 153 (2006), no. $12,2047-2054$

[43] P. Storm, The Novikov conjecture for mapping class groups as a corollary of Hamenstadt's theorem Preprint, available on-line at http://arxiv.org/abs/math.GT/0504248 (2005).

[44] J. Świątkowski, On the asymptotic homological dimension of hyperbolic groups, Bull. London Math. Soc. 27 (1995), no. 3, 209-221.

[45] J.-L. Tu, Remarks on Yu's "property A" for discrete metric spaces and groups, Bull. Soc. Math. France 129 (2001), no. 1, 115-139.

[46] S. Wolpert Geometry of Weil-Petersson completion of Teichmüller space, in Surveys in Differential Geometry, VIII: Papers in Honor of Calabi, Lawson, Siu and Uhlenbeck, (S. T. Yau, ed.) International Press, 2003.

[47] G. Yu, The Novikov conjecture for groups with finite asymptotic dimension, Annals of Mathematics 147 (1998), no. 2, 325-355.

[48] G. Yu, The coarse Baum-Connes conjecture for spaces which admit a uniform embedding into a Hilbert space, Invent. Math. 139 (2000), 201-240.

Mathematics and Statistics, UnC Greensboro, Greensboro, NC 27402, USA

E-mail address: gcbell@uncg.edu

Mathematical Institute, Tohoku University, Sendai, 980-8578 Japan

E-mail address: fujiwara@math.tohoku.ac.jp 